\title{
Multi-Epoch VLBA Observations of the SiO Maser Emission To- wards R Aquarii (R Aqr)
}

\author{
D. A. Boboltz, P. J. Diamond, \& A. J. Kemball \\ National Radio Astronomy Observatory, Socorro, NM 87801, U.S.A.
}

Abstract. We have observed $v=1, J=1-0,43-\mathrm{GHz}$ SiO maser emission towards the symbiotic Mira variable RAqr at four different epochs in the stellar pulsation cycle using the VLBA.

\section{Introduction}

Silicon monoxide ( $\mathrm{SiO}$ ) maser emission has been observed towards many latetype stars. The conditions necessary for the formation of $\mathrm{SiO}$ masers dictate that they be produced in a region which is inside the silicate dust formation point close to the surface of the star. Very Long Baseline Interferometry (VLBI) has shown that these masers do indeed lie close to the stellar surface at a distance of a $\sim 2-4 R_{*}$ (Diamond et al. 1994). This extended atmosphere is a complex region dominated by stellar pulsations and permeated by circumstellar shocks.

\section{Structure and Proper Motions}

The $\mathrm{SiO}$ masers towards $\mathrm{R}$ Aqr have been found to exhibit a ring-like morphology $\sim 31$ mas $(\sim 6.8 \mathrm{AU})$ in diameter. This ring of emission lies outside the stellar photosphere and within the circumstellar dust shell. The emission changes significantly over a time period of $\sim 1-2$ months with almost no similarity in structure on timescales $>6$ months. An analysis of the four epochs of observations has provided the first direct evidence of $\mathrm{SiO}$ maser proper motions. These observations, taken as the Mira variable approached maximum light, show that over a 98-day period the masers have an average inward proper motion of $\sim 1$ mas. This contraction of the maser shell implies an infall velocity of $\sim 4 \mathrm{~km} \mathrm{~s}^{-1}$ for the $\mathrm{SiO}$ masers during this phase of the stellar pulsation cycle.

\section{Polarization}

Images of both the linear and circular polarization structure were also produced for the $\mathrm{SiO}$ emission towards $\mathrm{R} \mathrm{Aqr}$. Maps of the maser component polarization are shown in Figure 1. These maps demonstrate that the $\mathrm{SiO}$ masers are significantly polarized, and that the polarization structure and intensity change on timescales as short as $\sim 1-2$ months. For three of the four epochs, a mean fractional circular polarization of $\sim 4 \%$ was determined implying a magnetic field strength $B \sec \theta \approx 13 \mathrm{G}$. For one of the four epochs, the mean fractional circular polarization was found to be $\sim 14 \%$ indicating a magnetic field strength $B \sec \theta \approx 46 \mathrm{G}$. The fractional linear polarization is fairly constant for all four epochs with mean values ranging from $20.8-25.0 \%$, and peak values as high as $\sim 83 \%$ for isolated maser features. Maps of the linear polarization vectors show an orderly structure over large portions of the maser shell indicating a uniform 


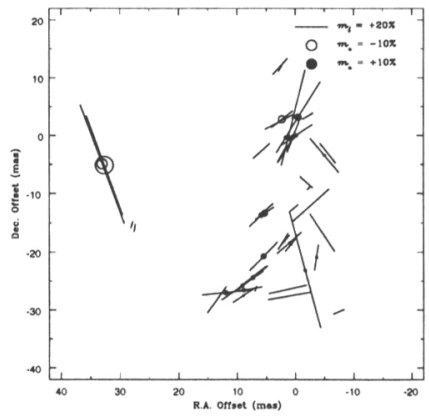

(a) Epoch 1 (30 June 1995, $\phi=0.31$ )

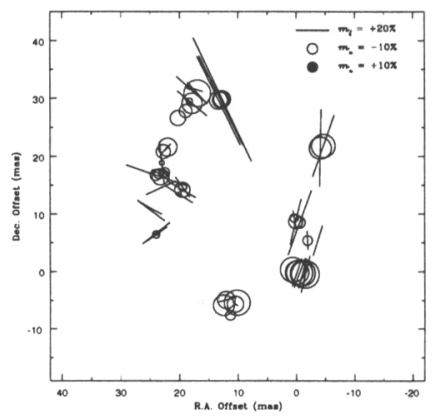

(c) Epoch 3 (2 Feb. 1996, $\phi=0.87$ )

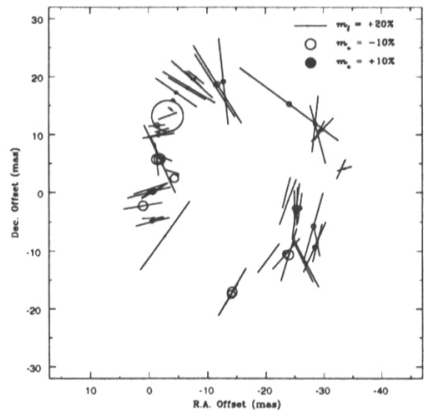

(b) Epoch 2 (29 Dec. 1995, $\phi=0.78$ )

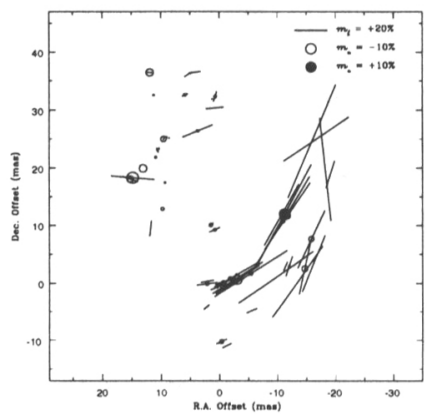

(d) Epoch 4 (5 April 1996, $\phi=0.04)$

Figure 1. Distribution of polarized components for Epochs 1-4. Components are plotted with a peak total intensity greater than $400 \mathrm{mJy}$. The radius of each circle is proportional to the degree of circular polarization $m_{c}$. Open circles denote negative sense of circular polarization while filled circles denote positive circular polarization. The degree of linear polarization $m_{l}$ is represented by the length of the line segments which are plotted at the polarization position angle $\chi$ for the component.

magnetic field topology in these regions of the extended atmosphere of $R$ Aqr. This uniform polarization structure is consistent with previous VLBI observations of the isolated Mira variable TX Cam (Kemball \& Diamond 1997).

Acknowledgments. DAB wishes to acknowledge the support of the NRAO pre-doctoral fellowship program. The National Radio Astronomy Observatory is a facility of the National Science Foundation, operated under a cooperative agreement by Associated Universities, Inc.

\section{References}

Diamond, P. J., et al, 1994. ApJ, 430, L61-64.

Kemball, A. J. \& Diamond, P. J. 1997. $A p J, 481$, L111-114. 